\title{
Dengue: defining protective versus pathologic immunity
}

\author{
Alan L. Rothman \\ Center for Infectious Disease and Vaccine Research, University of Massachusetts Medical School, Worcester, Massachusetts, USA.
}

\begin{abstract}
Dengue is an expanding public health problem, and an effective vaccine remains elusive. This review discusses how the significant influence of sequential infection with different dengue virus serotypes on the severity of disease can be viewed in terms of beneficial and detrimental effects of heterologous immunity. A more complete understanding of these effects is likely to be critical for predicting optimal vaccine-induced immune responses.
\end{abstract}

Among scientists in the developed world, there has been a recent resurgence of interest in exotic diseases, with the aim of protecting the global population from emerging infectious disease threats. The renewed commitment of effort and resources has welcome implications for the developing world, whose population faces the greatest part of these threats. Among the biological threats considered most serious are the viral hemorrhagic fevers. Although this term usually brings to mind the deadly outbreaks of Ebola virus, in reality over $99 \%$ of the cases of viral hemorrhagic fever reported worldwide are related instead to dengue hemorrhagic fever (DHF) (1).

DHF is caused by the dengue viruses (DENVs), members of the Flaviviridae family of small enveloped viruses (2). The flaviviruses carry a single-stranded RNA genome of relatively simple organization. A single open reading frame in the RNA directs the synthesis of a long polyprotein that is processed by viral and host cell proteases to produce the ten viral proteins, including three structural proteins (core [C]; membrane [M], produced as a precursor protein; and envelope [E]) and seven nonstructural (NS) proteins (Figure 1). The DENV complex encompasses four closely related serotypes: DENV-1, DENV-2, DENV-3, and DENV-4. All four DENV serotypes are transmitted between humans in nature by mosquitoes of the genus Aedes, principally Aedes aegypti, which is highly domesticated and has a preference for biting humans.

It has been estimated that over 50 million DENV infections occur globally each year (3). Most of these infections are clinically inapparent. Among symptomatic cases, the majority of subjects experience uncomplicated dengue fever (DF), an acute febrile illness typically lasting 3-7 days, accompanied by headache, myalgias, and, less often, a maculopapular rash. The headache and myalgias may be quite debilitating, which originated the name "break-bone fever" that was recorded prior to the 1900s (4). Laboratory findings in patients with DF include leukopenia thrombocytopenia, and mild elevations in serum hepatic transaminases (5). Fatigue may be prolonged for months after resolution of fever, but patients eventually recover without sequelae. None of these features is sufficiently specific for accurate clinical diagnosis of DF. Laboratory support for detection of IgM antibody or virus (by RT-PCR or virus isolation) is therefore important for recognition of outbreaks.

Nonstandard abbreviations used: antibody-dependent enhancement of infection (ADE); core (C); dengue fever (DF); dengue hemorrhagic fever (DHF); dengue virus (DENV); envelope (E); membrane (M); nonstructural (NS).

Conflict of interest: The author has declared that no conflict of interest exists.

Citation for this article: J. Clin. Invest. 113:946-951 (2004).

doi:10.1172/JCI200421512.
DHF represents the severe clinical manifestation of DENV infection, which occurs in no more than $3 \%$ of infected individuals (6). Fever, headache, and myalgias are prominent symptoms in DHF, as they are in DF. DHF is distinguished from DF on clinical grounds, with the three primary criteria being the occurrence of a vascular permeability defect resulting in plasma leakage; multifactorial hemostatic abnormalities, including marked thrombocytopenia; and a bleeding diathesis (7). Plasma leakage is evidenced by hemoconcentration and/or effusions in the pleural or peritoneal spaces, which usually occur after 3-5 days of fever, near the time of defervescence. When severe, the plasma leakage leads to hypotension and circulatory collapse, and this is the most important concern for triage and therapy. Thrombocytopenia is quite severe in DHF, with a platelet count of less than 100,000 cells $/ \mathrm{mm}^{3}$ required to fulfill the case definition. The nadir of the platelet count is typically coincident with plasma leakage, and severe thrombocytopenia also occurs in a significant percentage of patients with DF; nevertheless, the decline in platelet count typically precedes plasma leakage, and this finding has been useful in deciding whether to hospitalize patients suspected to have DHF (8). Bleeding manifestations, for example, from the gastrointestinal tract, can be severe in patients with DHF but are not usually severe enough to require transfusion and are rarely the principal cause of hypotension. Despite the use of the term "hemorrhagic fever," many patients with DHF have minor hemorrhagic manifestations; often the occurrence of petechiae elicited by inflation of a blood pressure cuff on the arm for $5 \mathrm{~min}$ utes (the "tourniquet test") is the only clinical sign.

The emergence of DHF as a global infectious disease threat is striking in its persistence and magnitude. Southeast Asia and the western Pacific region, where DHF was first recognized as a clinical entity, have faced this health problem for almost 50 years. Nevertheless, during each decade the number of DHF cases, the number of countries affected, and the geographic distribution of DHF have all increased steadily (3). DHF is, in comparison, a new problem in the tropical Western hemisphere, but one that has rapidly expanded in scope. Although essentially absent from the area prior to 1981, DHF has during the past decade been seen throughout Latin America and the Caribbean. It is currently estimated that over 3 billion individuals live in areas at risk for DHF and that hundreds of thousands of cases and thousands of deaths due to DHF occur each year, representing an urgent health problem to be addressed.

Human activities that have led to enhanced mosquito breeding capacity, increased interaction between mosquitoes and humans, and increased dispersal of viruses in both mosquito and human hosts have contributed (and continue to contribute) to the emer- 

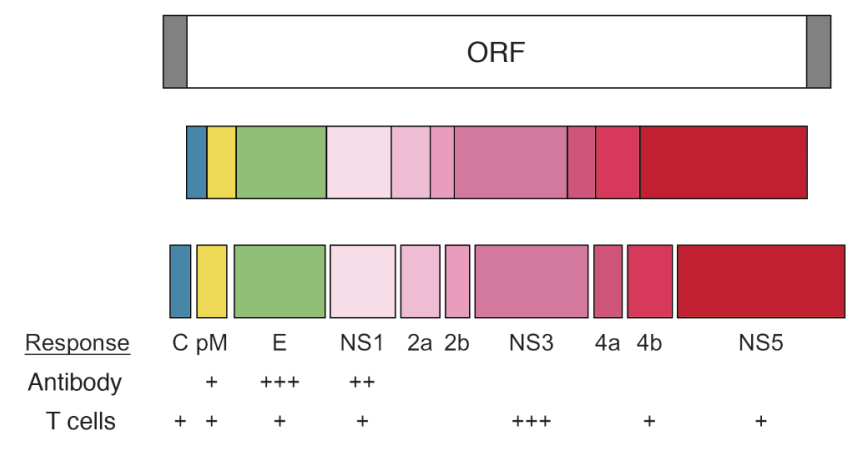

Figure 1

Organization of the flavivirus genome and its resulting proteins and the location of the major targets of the immune response. The DENV genome is a single-stranded sense RNA with a single open reading frame (ORF; top). The ORF is translated as a single polyprotein (middle) that is cleaved by viral and host proteases to yield the ten viral proteins: the $C$ protein; the $M$ protein, which is synthesized as the larger precursor protein pre-M (pM); the major E glycoprotein; and seven NS proteins involved in viral replication among other functions (bottom). The strength of the antibody and T cell responses to individual viral proteins is indicated below.

gence of DHF, as discussed in several recent reviews $(9,10)$. Poorly planned development has been a major factor, especially in urban centers of the developing world. The attendant inadequate sanitation and potable water supply, leading to an increase in mosquito breeding places, and the concentration of susceptible human hosts in close proximity to the mosquitoes also facilitate transmission. In the Western hemisphere, A. aegypti have also taken advantage of the lapse in mosquito control efforts that has occurred since the 1970s and have re-established essentially the full geographical range they commanded prior to the yellow fever eradication efforts of the mid-twentieth century.

At present, there is no specific therapy available for DHF. Appropriate fluid management to correct hypovolemia has been successful in reducing the mortality of $\operatorname{DHF}(7,11,12)$, but access to medical services remains problematic in many developing countries. Mosquito control, which is costly and often ineffective, remains the only method of preventing DHF currently available. Development of an effective vaccine against DENVs has therefore been considered a high priority by the WHO.

\section{Background of dengue vaccine development}

In principle, an effective vaccine against DENV is highly feasible. Unlike the situation with HIV and hepatitis C virus, DENV does not cause chronic infection. Viral replication is effectively controlled after a short (3- to 7-day) period of viremia, and individuals who have recovered from DENV infection are immune to rechallenge (13). Studies conducted in the 1940s demonstrated that a DENV strain derived by serial propagation in suckling mouse brain was attenuated in humans and could elicit protective immunity against DENV challenge (13).

There are a number of candidate DENV vaccines currently in development (Table 1) (14). Live attenuated strains of all four DENV serotypes have been derived by the traditional approach of serial propagation in primary dog or monkey kidney cells (15-17). This effort has been slowed by the lack of an animal model or in vitro markers of attenuation in humans. Improved molecular virology techniques and an improved understanding of the genomic structure of DENV have permitted a more rapid approach based on engineering of attenuating mutations into infectious cDNA clones of each of the four DENV serotypes, with the added theoretical advantage of a lower possibility of reversion to virulence (18-20). Another molecular approach being utilized is the creation of four separate infectious chimeric flaviviruses, each of which contains the pre-M and $\mathrm{E}$ genes of one of the four DENV serotypes in a single "backbone" containing the $\mathrm{C}$ and NS proteins of an attenuated flavivirus, either the yellow fever vaccine strain or an attenuated DENV strain $(21,22)$. DNA vaccines consisting of plasmids expressing one or a few proteins from each DENV serotype are in an earlier stage of development, as are subunit vaccines based on purified recombinant DENV proteins $(23,24)$. Several of these approaches have demonstrated protective efficacy in animal models of DENV infection, and a few have shown safety and immunogenicity in early phase clinical studies $(14,18,25,26)$.

Two main obstacles have delayed further clinical development of these candidate vaccines. First, an effective vaccine needs to prevent infection with all four DENV serotypes. Natural DENV infection induces long-lasting protective immunity only to the same serotype and only short-term (months) protection from infection with other serotypes (13). Although live attenuated and recombinant vaccines to all four DENV serotypes have been developed, incorporation of these into a tetravalent formulation that retains the immunogenicity of all four components has proven difficult, requiring the use of more complicated, multiple-dose immunization regimens (25).

The second and more significant obstacle is the current inability to predict whether candidate DENV vaccines will be at all effective in preventing DHF. DHF was unrecognized prior to the late 1950s. Therefore, the experimental challenge studies in humans conducted during and shortly after World War II did not attempt to test the efficacy of candidate vaccines against DHF. Subsequent studies of candidate vaccines have analyzed efficacy only in experimental ani-

\section{Table 1}

Immunological considerations and current status in development of candidate dengue vaccines

\begin{tabular}{lcccc}
\hline & Live attenuated & Chimeric virus & Plasmid DNA & Subunit \\
No. of dengue antigens included & All (10) & (10 for viral "backbone" only) & 1 to several & Usually 1 \\
In vivo replication & Yes & Nes & No \\
Elicits T and B cell memory & Best & Best & Excellent & Fair: Th, B; Poor: CTL \\
Anticipated durability of immune response & Best & Best & Good & Usually poor \\
Protective in animal models & Yes & Yes & Yes & Yes \\
Status of vaccine development & Phase I/II & Phase I/II & Preclinical & Preclinical \\
\hline
\end{tabular}


mal models, none of which faithfully reproduce the DHF syndrome seen in humans. As a result, selection of the most promising DENV vaccine candidates will necessarily rely on comparing vaccineinduced immune responses to a profile of protective immunity developed from observation of natural DENV infections.

\section{Immune responses to DENV}

The principal targets of the immune response to DENV are illustrated in Figure 1. The E glycoprotein is the principal component of the external surface of the DENV virion (27) and is a dominant target of the response consisting of antibodies against DENV. Antibodies against $\mathrm{E}$ have been shown to inhibit viral binding to cells and to neutralize viral infectivity in vitro (28). Passive transfer of antibodies against E protected mice from DENV challenge (29). Antibodies against E show variable degrees of cross-reactivity among the DENV serotypes, although neutralization of infectivity by antibodies is usually more serotype specific than virion binding (28). Binding of antibody to virus at non-neutralizing epitopes, or at concentrations below the neutralization endpoint, can enhance the uptake of virions into monocytic cell lines and primary human monocytes in vitro through interaction with cell surface Ig receptors, a phenomenon known as antibody-dependent enhancement of infection (ADE) (30). These "enhancing" antibodies do not increase infection of human DCs, which are highly permissive to DENV infection, however $(31,32)$.

While not a component of the virion, the NS1 protein is also an important target of antibodies against DENV. NS1 is expressed on the surface of infected cells and is also secreted into the circulation as a soluble multimer (33). Antibodies against NS1 can trigger complement-mediated lysis of DENV-infected cells in vitro and have been shown to protect mice from DENV challenge (34). A monoclonal antibody directed against the pre-M protein has also been shown to protect mice from DENV challenge (35); however, the mechanism of this protection and its relevance to natural protective immunity are uncertain.

As with other viruses, the $\mathrm{CD}^{+}$and $\mathrm{CD}^{+} \mathrm{T}$ cell response to DENV is directed against multiple viral proteins (Figure 1), although the NS3 protein appears to be particularly immunogenic, with a preponderance of the $\mathrm{T}$ cell epitopes identified $(36,37)$. DENV-reactive $T$ cells vary in their ability to recognize different DENV serotypes, depending upon the degree of homology at a given epitope. However, cross-reactivity with multiple serotypes is common, especially at epitopes in the more highly conserved NS proteins $(38,39)$. DENV-reactive $\mathrm{CD}^{+}$and $\mathrm{CD}^{+} \mathrm{T}$ cells predominantly produce high levels of IFN- $\gamma$ as well as TNF- $\alpha$, TNF- $\beta$, and chemokines including macrophage inhibitory protein- $1 \beta$ upon interaction with DENV-infected antigen presenting cells, and are efficient at lysis of DENV-infected cells in vitro $(39,40)$.

The extent to which these immune responses contribute to the long-term protective immunity afforded by natural primary DENV infection has not been fully defined. Symptomatic DENV infections, including cases of DHF, can occur despite the presence of antibodies capable of neutralizing in vitro infection of epithelial cell lines (41, 42). Neutralization of infection in monocytic cells (instead of enhancement of infection) may be more strongly associated with protection from DHF (41), although this association has been reported in only one study. There is very little published information on the association of specific $\mathrm{T}$ cell responses with protection. However, broadly serotype-cross-reactive IFN- $\gamma$ production in vitro showed a weak association with mild disease in a small study cohort (43).

\section{Evidence of an immunological basis for DHF}

The principal mechanisms by which DENV infection causes DHF have been a subject of controversy from the time the syndrome was first recognized. Opposing views have focused on the effect of viral and host factors on disease severity $(44,45)$.

Differences in virulence between naturally circulating DENV strains had been suspected based on differences in clinical profiles observed during isolated DENV outbreaks in Indonesia and the Pacific Islands $(46,47)$. More recently, studies in Peru and Sri Lanka have provided more convincing data, demonstrating the association of DHF with specific viral genotypes and not with others (48-50). Specific genetic determinants potentially explaining this association have been mapped to the E gene and to the $5^{\prime}$ and 3 ' untranslated regions of the DENV genome (51-53). However, in the absence of a validated model of virulence in humans, it has not been possible to verify that these genetic elements are responsible for virulence or to confirm any proposed mechanism for virulence.

Key seroepidemiological studies by Halstead and colleagues in Thailand during the 1960s first suggested an association of increased risk for DHF with a secondary DENV infection; that is, a new DENV infection in an individual who had previously experienced one or more DENV infections $(54,55)$. This hypothesis has since received strong confirmation from the "experiment of nature" in a large outbreak of DHF associated with DENV-2 in Cuba (56), as well as several independent prospective cohort studies in Southeast Asia (57-59). These observations have provided the basis for exploration of the possibility of a pathologic role for the immune response to DENV in the development of DHF. Age, race/ethnicity, nutritional state, and underlying chronic diseases such as asthma have also been suggested to contribute to DHF risk, although the data supporting these associations are weaker and the mechanisms involved are poorly defined.

Antibodies against DENV can affect the course of disease through multiple mechanisms (Table 2). Passive transfer of antibody against DENV did increase viremia titers in nonhuman primates in one study (60), and recent studies have demonstrated a positive correlation between peak viremia titer and disease severity in humans (61, 62 ), supporting the idea of the potential in vivo importance of ADE. The occurrence of DHF during primary DENV infection in the first year of life in children born to DENV-immune mothers, and who therefore acquire antibody against DENV transplacentally, also supports the idea of an in vivo role for $\operatorname{ADE}(63)$. However, high viremia titers in older children and adults with primary DENV infections and in clinically mild secondary DENV infections indicate that other factors are involved $(64,65)$. Immune complex formation in vivo has been detected in association with complement activation in patients with severe disease (66). Cross-reactivity of antibodies against $\mathrm{E}$ with plasminogen has been associated with bleeding in acute DENV infection, although not with DHF (67).

Cytokine production and cytolysis by activated $\mathrm{T}$ lymphocytes are additional potential contributors to disease (Table 2). Elevations of circulating levels of activation markers including soluble TNF receptors, soluble IL-2 receptors, and soluble CD8 have been shown to correlate with disease severity $(61,68-71)$. Similar associations with disease severity have been shown for the expression of activation markers on circulating $\mathrm{CD}^{+} \mathrm{T}$ cells $(72)$ and the expansion of DENV epitope-specific T cell populations $(73,74)$. Increased production of various cytokines, including IFN- $\gamma$, TNF- $\alpha$, IL-10, and chemokines has also been detected in acute DENV infection (69-71, 75-77). Although elevations in both type 1 and type 2 cytokine lev- 


\section{Table 2}

Postulated pathologic mechanisms of DENV-reactive immune responses in the development of DHF

Mechanism
Antibodies
Enhancement of infection
Immune complex formation
Cross-reactivity to coagulation and endothelial cell proteins
T lymphocytes
Proinflammatory cytokine production
Lysis of bystander (uninfected) cells

Mechanism

Immune complex formatio

Cross-reactivity to coagulation and endothelial cell proteins

Proinflammatory cytokine production

\author{
Postulated effect(s) \\ Increased cellular infection, increased viral burden \\ Complement activation \\ Bleeding, endothelial cell dysfunction \\ Increased vascular permeability \\ Hepatic injury
}

References

$(45,60,84)$

$(66,85,86)$

$(67,87,88)$

$(61,68,69)$

(40) els are detected in DHF, the timing of their production appears to be an important factor with earlier induction of type 1 cytokines being associated with more severe disease (61). Analysis of T cell responses to DENV prior to secondary DENV infections showed an association between in vitro TNF- $\alpha$ responses to DENV antigens and more severe disease during the subsequent infection (43).

\section{Heterologous immunity and the pathogenesis of DHF}

Although similar response mechanisms appear to operate in both protective and pathological immunity to DENV, a principal immunological consideration is the heterologous nature of secondary DENV infection. The DENV causing secondary disease is always of a different serotype than the virus that induced immune responses during the earlier DENV infection, and therefore the antibodies and memory $\mathrm{T}$ lymphocytes induced by the primary DENV infection typically encounter antigens that differ (although some may be the same) in sequence from their original target antigen. The magnitude of these sequence differences is epitope dependent, but all sequence differences have the potential to affect the quality of the effector response and also modify the immunological repertoire. We speculate that the immune response to these heterologous sequences has the net effect of altering the balance between a protective and pathological outcome.

The effect of sequence differences at antibody-binding sites is to reduce the avidity of the interactions between the pre-existing antibodies and the new DENV serotype. These less avid interactions have a significant effect on the ability of the antibodies to neutralize virus infectivity. However, there is sufficient binding of antibody to the virion to trigger $\mathrm{ADE}$. In addition, these low-avidity antibodies increase in titer in preference to new antibodies with high avidity for the new DENV serotype, because the pre-existing memory B cells and plasma cells are more rapidly activated than naive B cells. The pattern of antibody responses in convalescence, therefore, is influenced heavily by the serotype of the primary DENV infection, a phenomenon referred to as "original antigenic sin" (78).

The effect of sequence differences at $T$ cell epitopes is more complex, consistent with the wider range of effector functions of $\mathrm{T}$ cells. Variant peptides, or altered peptide ligands, induce different activation signals in antigen-specific $\mathrm{T}$ lymphocytes and thereby modulate specific effector functions of $\mathrm{CD}^{+}$and $\mathrm{CD}^{+} \mathrm{T}$ cells $(79$, $80)$. In general, such partial $\mathrm{T}$ cell agonists have the greatest effect on the proliferation responses and less effect on cytotoxicity responses. Some partial T cell agonists fail to induce cytokine production, whereas others induce an altered cytokine response profile. We have observed just such effects in the in vitro responses of DENV-reactive T cells to peptides of heterologous DENV serotypes
(81). Furthermore, a phenomenon analogous to "original antigenic sin" would be expected to occur in the T cell response to secondary DENV infections, in which expansion of the pre-existing lower avidity memory $\mathrm{T}$ cell populations takes precedence over the expansion of naive $T$ cell populations with higher avidity for the new DENV serotype. Recent studies of the $T$ cell repertoire after secondary DENV infection support this view (74).

The potential importance of heterologous immunity in influencing the severity of DENV disease is bolstered by data from an animal model of sequential virus infections. In this experimental system, previous infection with one virus (e.g., lymphocytic choriomeningitis virus) altered the response to a subsequent vaccinia virus challenge, altering the hierarchy of epitope-specific $\mathrm{T}$ cell responses and resulting in lower virus titers but greater tissue

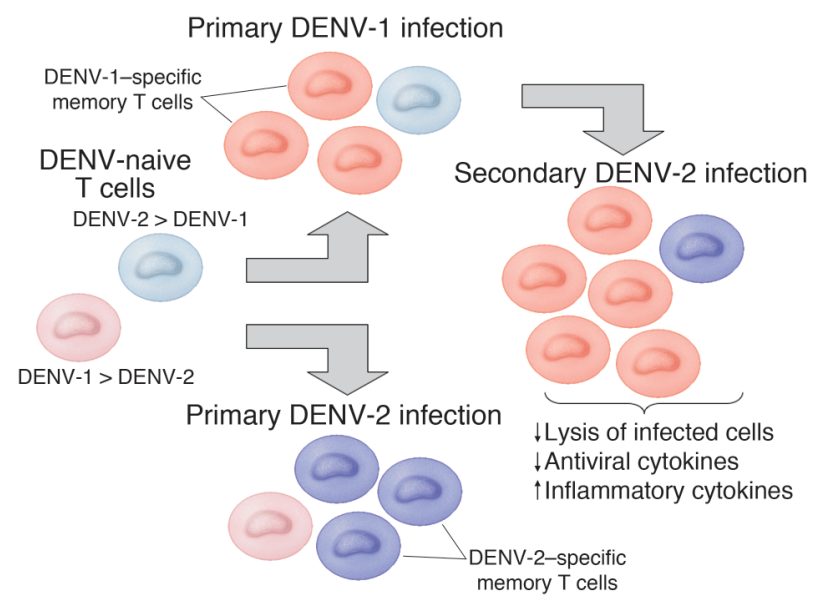

Figure 2

Proposed model of heterologous immunity in secondary dengue virus infections and its implications for the pathogenesis of dengue hemorrhagic fever. Primary DENV-2 infection and sequential DENV-1 and DENV-2 infections are compared for illustration purposes. The naive $T$ cell repertoire (pale colors) likely contains some cells with higher avidity for DENV-1 than DENV-2 (red; DENV-1 > DENV-2) and other cells with higher avidity for DENV-2 than DENV-1 (blue; DENV-2 > DENV-1). During primary infection, $T$ cell populations with higher avidity for the infecting serotype are preferentially expanded and enter the memory pool (shown as darker colors). When DENV-2 infection follows DENV-1 infection, the memory $T$ cell populations with higher avidity for the earlier infection expand more rapidly than do naive $T$ cell populations. Because these DENV-1-specific memory T cells have lower avidity for DENV-2, viral clearance mechanisms are suboptimal, whereas proinflammatory responses contribute to disease. 
inflammation $(82,83)$. A model for the effect of heterologous immunity in the pathogenesis of DHF is shown in Figure 2. We postulate that the low-avidity $\mathrm{T}$ cells that dominate the response to secondary DENV infections are less than optimally efficient at elimination of DENV-infected cells and that the pattern of cytokine production is altered such that proinflammatory cytokine production (e.g., TNF- $\alpha$ ) is enhanced and antiviral cytokine production (e.g., IFN- $\gamma$ ) is reduced. These effects could combine to induce the plasma leakage symptomatic of DHF.

\section{Responding to the challenge}

To maximize protection and minimize pathologic heterologous immunity and achieve long-lasting immune responses, an optimal vaccine to prevent DENV infections including DHF should induce high-avidity antibodies and $\mathrm{T}$ lymphocytes against all four DENV serotypes. The theoretical advantages of live attenuated virus vaccines or plasmid DNA vaccines (Table 1 ) have placed them on a faster track toward clinical development. Tetravalent attenuated vaccines have been developed, but researchers have encountered problems in finding a formulation that balances the immunogenicity of all four viruses (25). Recombinant chimeric flavivirus vaccines show promise in induction of antibodies against $\mathrm{E}$ (21), but it will be important to determine whether the common epitopes in the NS proteins of the viral "backbone" will induce optimal T cell responses. Some findings suggest that individuals with mild and severe disease target different epitopes in their $\mathrm{T}$ cell response to DENV (73). Nucleotide sequences from different DENVs could theoretically be introduced into a single vaccine "backbone" to provide the most favorable targets for a high-avidity, cross-serotype immune response. Much additional research is needed to identify and characterize protective and pathological immune responses in order to make an effective DENV vaccine a reality.

Address correspondence to: Alan L. Rothman, Center for Infectious Disease and Vaccine Research, Room S5-326, University of Massachusetts Medical School, 55 Lake Avenue North, Worcester, Massachusetts 01655, USA. Phone: (508) 856-4182; Fax: (508) 8564890; E-mail: alan.rothman@umassmed.edu.
1. Rigau-Perez, J.G., et al. 1998. Dengue and dengue haemorrhagic fever. Lancet. 352:971-977.

2. Henchal, E.A., and Putnak, J.R. 1990. The dengue viruses. Clin. Microbiol. Rev. 3:376-396.

3. Pinheiro, F.P., and Corber, S.J. 1997. Global situation of dengue and dengue haemorrhagic fever, and its emergence in the Americas. World Health Stat. Q. 50:161-169.

4. Rigau-Perez, J.G. 1998. The early use of break-bone fever (Quebranta huesos, 1771) and dengue (1801) in Spanish. Am. J. Trop. Med. Hyg. 59:272-274.

5. Kalayanarooj, S., et al. 1997. Early clinical and laboratory indicators of acute dengue illness. J. Infect. Dis. 176:313-321.

6. Halstead, S.B. 1980. Immunological parameters of togavirus disease syndromes. In The togaviruses. biology, structure, replication. R.W. Schlesinger, editor. Academic Press. New York, New York, USA. 107-173.

7. 1997. Dengue haemorrhagic fever: diagnosis, treatment and control. World Health Organization. Geneva, Switzerland. 1-58.

8. Chin, C.K., et al. 1993. Protocol for out-patient management of dengue illness in young adults. J. Trop. Med. Hyg. 96:259-263.

9. Gubler, D.J., and Clark, G.G. 1995. Dengue/dengue hemorrhagic fever: the emergence of a global health problem. Emerg. Infect. Dis. 1:55.

10. Pinheiro, F.P., and Chuit, R. 1998. Emergence of dengue hemorrhagic fever in the Americas. Infect. Med. 15:244-251.

11. Ngo, N.T., et al. 2001. Acute management of dengue shock syndrome: a randomized doubleblind comparison of 4 intravenous fluid regimens in the first hour. Clin. Infect. Dis. 32:204-213.

12. Harris, E., et al. 2003. Fluid intake and decreased risk for hospitalization for dengue fever, Nicaragua. Emerg. Infect. Dis. 9:1003-1006.

13. Sabin, A.B. 1952. Research on dengue during World War II. Am. J. Trop. Med. Hyg. 1:30-50.

14. Barrett, A.D. 2001. Current status of flavivirus vaccines. Ann. N. Y. Acad. Sci. 951:262-271.

15. Bhamarapravati, N., and Sutee, Y. 2000. Live attenuated tetravalent dengue vaccine. Vaccine. 18:44-47.

16. Eckels, K.H., et al. 2003. Modification of dengue virus strains by passage in primary dog kidney cells: preparation of candidate vaccines and immunization of monkeys. Am. J. Trop. Med. Hyg. 69:12-16.

17. Innis, B.L., and Eckels, K.H. 2003. Progress in development of a live-attenuated, tetravalent dengue virus vaccine by the United States Army Medical Research and Materiel Command. Am.J. Trop. Med. Hyg. 69:1-4.

18. Durbin, A.P., et al. 2001. Attenuation and immuno- genicity in humans of a live dengue virus type- 4 vaccine candidate with a 30 nucleotide deletion in its 3'-untranslated region. Am. J. Trop. Med. Hyg. 65:405-413.

19. Markoff, L., et al. 2002. Derivation and characterization of a dengue type 1 host range-restricted mutant virus that is attenuated and highly immunogenic in monkeys. J. Virol. 76:3318-3328.

20. Blaney, J.E., Jr., Manipon, G.G., Murphy, B.R., and Whitehead, S.S. 2003. Temperature sensitive mutations in the genes encoding the NS1, NS2A, NS3, and NS5 nonstructural proteins of dengue virus type 4 restrict replication in the brains of mice. Arch. Virol. 148:999-1006.

21. Guirakhoo, F., et al. 2002. Viremia and immunogenicity in nonhuman primates of a tetravalent yellow fever-dengue chimeric vaccine: genetic reconstructions, dose adjustment, and antibody responses against wild-type dengue virus isolates. Virology. 298:146-159.

22. Huang, C.Y., et al. 2003. Dengue 2 PDK-53 virus as a chimeric carrier for tetravalent dengue vaccine development. J. Virol. 77:11436-11447.

23. Chang, G.J., Davis, B.S., Hunt, A.R., Holmes, D.A., and Kuno, G. 2001. Flavivirus DNA vaccines: current status and potential. Ann. N. Y. Acad. Sci. 951:272-285.

24. Simmons, M., Murphy, G.S., Kochel, T., Raviprakash, K., and Hayes, C.G. 2001. Characterization of antibody responses to combinations of a dengue-2 DNA and dengue-2 recombinant subunit vaccine. Am. J. Trop. Med. Hyg. 65:420-426.

25. Kanesa-thasan, N., et al. 2001. Safety and immunogenicity of attenuated dengue virus vaccines (Aventis Pasteur) in human volunteers. Vaccine. 19:3179-3188.

26. Sabchareon, A., et al. 2004. Safety and immunogenicity of a three dose regimen of two tetravalent live-attenuated dengue vaccines in five- to twelveyear-old Thai children. Pediatr. Infect. Dis. J. 23:99-109.

27. Kuhn, R.J., et al. 2002. Structure of dengue virus: implications for flavivirus organization, maturation, and fusion. Cell. 108:717-725.

28. Roehrig, J.T., Bolin, R.A., and Kelly, R.G. 1998. Monoclonal antibody mapping of the envelope glycoprotein of the dengue 2 virus, Jamaica. Virology. 246:317-328

29. Kaufman, B.M., Summers, P.L., Dubois, D.R., and Eckels, K.H. 1987. Monoclonal antibodies against dengue 2 virus E-glycoprotein protect mice against lethal dengue infection. Am. J. Trop. Med. Hyg. 36:427-434.

30. Morens, D.M., and Halstead, S.B. 1990. Measure- ment of antibody-dependent infection enhancement of four dengue virus serotypes by monoclonal and polyclonal antibodies. J. Gen. Virol. 71:2909-2914.

31. Wu, S.J., et al. 2000. Human skin Langerhans cells are targets of dengue virus infection. Nat. Med. 6:816-820.

32. Libraty, D.H., Pichyangkul, S., Ajariyakhajorn, C., Endy, T.P., and Ennis, F.A. 2001. Human dendritic cells are activated by dengue virus infection: enhancement by gamma interferon and implications for disease pathogenesis. J. Virol. 75:3501-3508.

33 Young, P.R., Hilditch, P.A., Bletchly, C., and Halloran, W. 200V0. An antigen capture enzyme-linked immunosorbent assay reveals high levels of the dengue virus protein NS1 in the sera of infected patients. J. Clin. Microbiol. 38:1053-1057.

34. Schlesinger, J.J., Brandriss, M.W., and Walsh, E.E. 1987. Protection of mice against dengue 2 virus encephalitis by immunization with the dengue 2 virus non-structural glycoprotein NS1.J. Gen. Virol. 68:853-857.

35. Kaufman, B.M., et al. 1989. Monoclonal antibodies for dengue virus prM glycoprotein protect mice against lethal dengue infection. Am. J. Trop. Med. Hyg. 41:576-580.

36. Kurane, I., Brinton, M.A., Samson, A.L., and Ennis, F.A. 1991. Dengue virus-specific, human $\mathrm{CD}^{+}$ CD8- cytotoxic T-cell clones: multiple patterns of virus cross-reactivity recognized by NS3-specific T-cell clones. J. Virol. 65:1823-1828.

37. Lobigs, M., Arthur, C.E., Mullbacher, A., and Blanden, R.V. 1994. The flavivirus nonstructural protein NS3 is a dominant source of cytotoxic T cell peptide determinants. Virology. 202:195-201.

38. Kurane, I., Zeng, L., Brinton, M.A., and Ennis, F.A. 1998. Definition of an epitope on NS3 recognized by human $\mathrm{CD}^{+}$cytotoxic $\mathrm{T}$ lymphocyte clones cross-reactive for dengue virus types 2,3 , and 4 . Virology. 240:169-174.

39. Zivny, J., et al. 1995. A single nine-amino acid peptide induces virus-specific, $\mathrm{CD}^{+}$human cytotoxic $\mathrm{T}$ lymphocyte clones of heterogenous serotype specificities. J. Exp. Med. 182:853-863.

40. Gagnon, S.J., Ennis, F.A., and Rothman, A.L. 1999. Bystander target cell lysis and cytokine production by dengue virus-specific human $\mathrm{CD} 4^{+}$cytotoxic $\mathrm{T}$ lymphocyte clones. J. Virol. 73:3623-3629.

41. Kliks, S.C., Nisalak, A., Brandt, W.E., Wahl, L., and Burke, D.S. 1989. Antibody-dependent enhancement of dengue virus growth in human monocytes as a risk factor for dengue hemorrhagic fever. Am. J. Trop. Med. Hyg. 40:444-451. 
42. Endy, T.P., et al. 2004. Relationship of preexisting dengue virus (DV) neutralizing antibody levels to viremia and severity of disease in a prospective cohort study of DV infection in Thailand. J. Infect. Dis. 189:990-1000.

43. Mangada, M.M., et al. 2002. Dengue-specific T cell responses in peripheral blood mononuclear cells obtained prior to secondary dengue virus infections in Thai schoolchildren. J. Infect. Dis. 185:1697-1703.

44. Rosen, L. 1977. The Emperor's New Clothes revisited, or reflections on the pathogenesis of dengue hemorrhagic fever. Am. J. Trop. Med. Hyg. 26:337-343.

45. Halstead, S.B. 1989. Antibody, macrophages, dengue virus infection, shock, and hemorrhage: a pathogenetic cascade. Rev. Infect. Dis. 11:S830-S839.

46. Gubler, D.J., Suharyono, W., Tan, R., Abidin, M., and Sie, A. 1981. Viraemia in patients with naturally acquired dengue infection. Bull. World Health Organ. 59:623-630.

47. Gubler, D.J., Suharyono, W., Lubis, I., Eram, S., and Gunarso, S. 1981. Epidemic dengue 3 in central Java, associated with low viremia in man. Am.J. Trop. Med. Hyg. 30:1094-1099.

48. Watts, D.M., et al. 1999. Failure of secondary infection with American genotype dengue 2 to cause dengue haemorrhagic fever. Lancet. 354:1431-1434.

49. Messer, W.B., et al. 2002. Epidemiology of dengue in Sri Lanka before and after the emergence of epidemic dengue hemorrhagic fever. Am. J. Trop. Med. Hyg. 66:765-773.

50. Messer, W.B., Gubler, D.J., Harris, E., Sivananthan, K., and de Silva, A.M. 2003. Emergence and global spread of a dengue serotype 3, subtype III virus. Emerg. Infect. Dis. 9:800-809.

51. Leitmeyer, K.C., et al. 1999. Dengue virus structural differences that correlate with pathogenesis. J. Virol. 73:4738-4747.

52. Pryor, M.J., et al. 2001. Replication of dengue virus type 2 in human monocyte-derived macrophages: comparisons of isolates and recombinant viruses with substitutions at amino acid 390 in the envelope glycoprotein. Am. J. Trop. Med. Hyg. 65:427-434

53. Cologna, R., and Rico-Hesse, R. 2003. American genotype structures decrease dengue virus output from human monocytes and dendritic cells. J. Virol. 77:3929-3938

54. Halstead, S.B., Nimmannitya, S., and Cohen, S.N. 1970. Observations related to pathogenesis of dengue hemorrhagic fever. IV. Relation of disease severity to antibody response and virus recovered. Yale J. Biol. Med. 42:311-328.

55. Halstead, S.B. 1970 . Observations related to pathogenesis of dengue hemorrhagic fever. VI. Hypotheses and discussion. Yale J. Biol. Med. 42:350-362.

56. Guzman, M.G., et al. 1990. Dengue hemorrhagic fever in Cuba, 1981: a retrospective seroepidemiologic study. Am. J. Trop. Med. Hyg. 42:179-184.

57. Sangkawibha, N., et al. 1984. Risk factors for dengue shock syndrome: a prospective epidemiologic study in Rayong, Thailand. I. The 1980 out- break. Am. J. Epidemiol. 120:653-669.

58. Burke, D.S., Nisalak, A., Johnson, D.E., and Scott, R.M. 1988. A prospective study of dengue infections in Bangkok. Am. J. Trop. Med. Hyg. 38:172-180.

59. Thein, S., et al. 1997. Risk factors in dengue shock syndrome. Am. J. Trop. Med. Hyg. 56:566-572.

60. Halstead, S.B. 1979. In vivo enhancement of dengue virus infection in rhesus monkeys by passively transferred antibody. J. Infect. Dis. 140:527-533.

61. Libraty, D.H., et al. 2002. Differing influences of viral burden and immune activation on disease severity in secondary dengue 3 virus infections. J. Infect. Dis. 185:1213-1221.

62. Murgue, B., Roche, C., Chungue, E., and Deparis, X. 2000. Prospective study of the duration and magnitude of viraemia in children hospitalised during the 1996-1997 dengue-2 outbreak in French Polynesia. J. Med. Virol. 60:432-438.

63. Kliks, S.C., Nimmanitya, S., Nisalak, A., and Burke, D.S. 1988. Evidence that maternal dengue antibodies are important in the development of dengue hemorrhagic fever in infants. Am. J. Trop. Med. Hyg. 38:411-419.

64. Sudiro, T.M., et al. 2001. Analysis of plasma viral RNA levels during acute dengue virus infection using quantitative competitor reverse transcription-polymerase chain reaction. J. Med. Virol. 63:29-34.

65. Vaughn, D.W., et al. 1997. Dengue in the early febrile phase: viremia and antibody responses. J. Infect. Dis. 176:322-330.

66. Theofilopoulos, A.N., Wilson, C.B., and Dixon, F.J. 1976. The Raji cell radioimmune assay for detecting immune complexes in human sera. J. Clin. Invest. 57:169-182.

67. Chungue, E., et al. 1994. Correlation between detection of plasminogen cross-reactive antibodies and hemorrhage in dengue virus infection. J. Infect. Dis. 170:1304-1307.

68. Kurane, I., et al. 1991. Activation of T lymphocytes in dengue virus infections. High levels of soluble interleukin 2 receptor, soluble CD4, soluble CD8, interleukin 2, and interferon-gamma in sera of children with dengue. J. Clin. Invest. 88:1473-1480.

69. Green, S., et al. 1999. Early immune activation in acute dengue is related to development of plasma leakage and disease severity. J. Infect. Dis. 179:755-762.

70. Hober, D., Delannoy, A.S., Benyoucef, S., De Groote, D., and Wattre, P. 1996. High levels of sTNFR P75 and TNF alpha in dengue-infected patients. Microbiol. Immunol. 40:569-573.

71. Bethell, D.B., et al. 1998. Pathophysiologic and prognostic role of cytokines in dengue hemorrhagic fever. J. Infect. Dis. 177:778-782.

72. Green, S., et al. 1999. Early CD69 expression on peripheral blood lymphocytes from children with dengue hemorrhagic fever. J. Infect. Dis. 180:1429-1435.

73. Zivna, I., et al. 2002. T cell responses to an HLA $B * 07$-restricted epitope on the dengue NS3 protein correlate with disease severity. J. Immunol.
168:5959-5965

74. Mongkolsapaya, J., et al. 2003. Original antigenic $\sin$ and apoptosis in the pathogenesis of dengue hemorrhagic fever. Nat. Med. 9:921-927.

75. Green, S., et al. 1999. Elevated plasma interleukin10 levels in acute dengue correlate with disease severity. J. Med. Virol. 59:329-334.

76. Mustafa, A.S., Elbishbishi, E.A., Agarwal, R., and Chaturvedi, U.C. 2001. Elevated levels of interleukin-13 and IL-18 in patients with dengue hemorrhagic fever. FEMS Immunol. Med. Microbiol. 30:229-233.

77. Hober, D., et al. 1993. Serum levels of tumor necrosis factor- $\alpha$ (TNF- $\alpha$ ), interleukin-6 (IL-6), and interleukin-1 $(\mathrm{IL}-1 \beta)$ in dengue-infected patients. $A m$. J. Trop. Med. Hyg. 48:324-331.

78. Halstead, S.B., Rojanasuphot, S., and Sangkawibha, N. 1983. Original antigenic sin in dengue. Am.J. Trop. Med. Hyg. 32:154-156.

79. Evavold, B.D., Sloan-Lancaster, J., and Allen, P.M. 1993. Tickling the TCR: selective T-cell functions stimulated by altered peptide ligands. Immunol. Today 14:602-609.

80. Sloan-Lancaster, J., and Allen, P.M. 1996. Altered peptide ligand-induced partial $\mathrm{T}$ cell activation: molecular mechanisms and role in $\mathrm{T}$ cell biology. Annu. Rev. Immunol. 14:1-27.

81. Zivny, J., et al. 1999. Partial agonist effect influences the CTL response to a heterologous dengue virus serotype. J. Immunol. 163:2754-2760.

82. Selin, L.K., Varga, S.M., Wong, I.C., and Welsh, R.M. 1998. Protective heterologous antiviral immunity and enhanced immunopathogenesis mediated by memory $\mathrm{T}$ cell populations. J. Exp. Med. 188:1705-1715.

83. Chen, H.D., Fraire, A.E., Joris, I., Welsh, R.M., and Selin, L.K. 2003. Specific history of heterologous virus infections determines anti-viral immunity and immunopathology in the lung. Am. J. Pathol. 163:1341-1355.

84. Morens, D.M. 1994. Antibody-dependent enhancement of infection and the pathogenesis of viral disease. Clin. Infect. Dis. 19:500-512.

85. Bokisch, V.A., Top, F.H., Jr., Russell, P.K., Dixon, F.J., and Muller-Eberhard, H.J. 1973. The potential pathogenic role of complement in dengue hemorrhagic shock syndrome. N. Engl. J. Med. 289:996-1000.

86. Malasit, P. 1987. Complement and dengue haemorrhagic fever/shock syndrome. SE Asian J. Trop. Med. Pub. Health. 18:316-320.

87. Falconar, A.K.I. 1997. The dengue virus nonstructural-1 protein (NS1) generates antibodies to common epitopes on human blood clotting, integrin/adhesion proteins and binds to human endothelial cells: potential implications in haemorrhagic fever pathogenesis. Arch. Virol. 142:897-916.

88. Markoff, L.J., Innis, B.L., Houghten, R., and Henchal, L.S. 1991. Development of cross-reactive antibodies to plasminogen during the immune response to dengue virus infection. J. Infect. Dis. 164:294-301. 